\title{
Role of levonorgestrel releasing intra-uterine system in the treatment of menorrhagia due to dysfunctional uterine bleeding and fibroid uterus
}

\author{
Reena Gupta ${ }^{1}$, Rupali Dewan ${ }^{1}$, Pratima Mittal', \\ Jyotsna Suri ${ }^{1}$, Abhinav Dewan ${ }^{2}$, Rekha Bharti ${ }^{1}$
}

\author{
${ }^{1}$ Department of Obstetrics \& Gynaecology, VMMC and Safdarjung Hospital, New Delhi-110029, India \\ ${ }^{2}$ Department of Radiation Oncology, Rajiv Gandhi Cancer Institute and Research Centre, Delhi, India
}

Received: 22 July 2014

Accepted: 8 August 2014

\author{
*Correspondence: \\ Dr. Reena Gupta, \\ E-mail: reenagupta17.8@gmail.com
}

(C) 2014 Gupta R et al. This is an open-access article distributed under the terms of the Creative Commons Attribution Non-Commercial License, which permits unrestricted non-commercial use, distribution, and reproduction in any medium, provided the original work is properly cited.

\begin{abstract}
Background: This study was carried out to assess the clinical effectiveness of levonorgestrel releasing intrauterine device (LNG-IUS) in the treatment of menorrhagia due to either Dysfunctional Uterine Bleeding (DUB) or fibroid in Indian patients, and to assess patient satisfaction with this treatment modality.

Methods: Sixty women with menorrhagia, 30 due to fibroid and 30 due to DUB, meeting inclusion criteria, received LNG-IUS and were prospectively followed up for 9 months with periodic clinical assessments and transvaginal ultrasounds. Patient satisfaction was assessed on a five-point scale.

Results: One patient in DUB group was lost to follow-up. In DUB patients, the treatment failure rate was only $3.4 \%$ (1 out of 29 patients). The median PBAC score reduced by $95 \%$ at 9 months. Fibroid patients also had an equally impressive $97.7 \%$ reduction of PBAC score at 9 months, although the failure rate was higher (23.3\%; 7 out of 30). Majority of patients were either 'very satisfied' or 'satisfied' with the treatment, although this percentage was more in DUB $(82.8 \%)$ than in the fibroid group $(66.7 \%)$. Haemoglobin and serum ferritin levels significantly increased in both groups. No major side effect was noted.

Conclusions: LNG-IUS is an excellent treatment modality for patients of DUB, with good patient satisfaction. It is also a useful treatment option in non-submucosal small fibroids for the symptoms of menorrhagia, can reduce uterine volume and can help avoid hysterectomy, but there is no effect on fibroid volume. Majority of patients are satisfied, although satisfaction rates are less than in DUB patients.
\end{abstract}

Keywords: Levonorgestrel releasing intrauterine device, Menorrhagia, Fibroid, Dysfunctional uterine bleeding

\section{INTRODUCTION}

Menorrhagia is an important cause of ill health in women worldwide. About one third of women report heavy menstrual bleeding at some point of time in their lives. Menorrhagia is defined as a menstrual blood loss of atleast $80 \mathrm{ml}$ per cycle. ${ }^{1}$ The heavy menstrual bleeding leads to discomfort, anxiety, poor quality of life, as well as medical problems like iron deficiency anaemia, especially in Indian women who already have low iron reserves. $^{2,3}$ It is the major symptom among majority of women who undergo hysterectomy. ${ }^{4,5}$

Menorrhagia can be caused by systemic, local or iatrogenic disorders. If women with menorrhagia have no pelvic or organic pathology then the diagnosis of 'dysfunctional uterine bleeding' is used. Systemic disorders comprise altered thyroid function and haemophilic conditions, whereas, local disorders include causes such as uterine leiomyomas, adenomyosis or polyps. 
Various treatment modalities are available for menorrhagia varying from medical to surgical. In cases of dysfunctional uterine bleeding and uterine fibroids, hysterectomy is more effective than other treatment modalities for the symptoms of menorrhagia but the associated morbidity and complications cannot be ignored. Concern over rising rates of hysterectomy and its associated complications has resulted in great interest in all surgical and medical treatments for menorrhagia, especially in women who want to preserve their uterus. Minimally invasive surgical techniques like endometrial ablation/resection are considered to be valid and cost effective alternatives, but require good hysteroscopic skills and are not without operative morbidity. Oral medical treatment is often not acceptable because the drug has to be taken daily for prolonged period which leads to poor compliance and systemic side effects.

One minimally invasive procedure for control of menorrhagia is the insertion of levonorgestrel releasing intrauterine device (LNG-IUS). In recent years LNG-IUS has been increasingly advocated as an alternative to hysterectomy for menorrhagia. ${ }^{6-10}$ This intrauterine system consists of a $\mathrm{T}$-shaped polyethylene frame (32 $\mathrm{mm} \times 32 \mathrm{~mm}$ ) with a levonorgestrel $(\mathrm{LNG})$-containing reservoir around its vertical stem. It contains a 1:1 mixture of $52 \mathrm{mg}$ of LNG and polydimethylsiloxane as the carrier polymer. It is effective for 5 years, and during this period, the cylinder releases, via a drug-contolling membrane, $20 \mu \mathrm{g}$ of LNG per 24 hours. ${ }^{11} \mathrm{LNG}$, delivered to the uterine cavity, is absorbed systemically, and maximum serum levels are reached within a few hours; systemic serum levels are maintained at around 150-200 $\mathrm{pg} / \mathrm{ml}^{12}$

LNG-IUS insertion results in immediate and profound effects upon endometrial morphology and function, with extensive decidualization of the endometrial stromal cells, atrophy of the glandular and surface epithelium and changes in vascular morphology. ${ }^{13}$ The immediate and intense suppression of endometrium can reduce both the duration of bleeding \& the amount of menstrual blood loss. ${ }^{14}$ In addition to decrease in menstrual blood loss, insertion of an LNG-IUS in women with myomas may result in a decrease in volume of myomas \& total uterine volume with no development of new myoma uterus. ${ }^{15}$

This study was carried out to assess the clinical effectiveness of levonorgestrel releasing intrauterine device (LNG-IUS) in the treatment of menorrhagia due to either dysfunctional uterine bleeding or fibroid uterus in Indian patients, and to assess patient satisfaction with this treatment modality.

\section{METHODS}

This was a prospective observational study conducted in the Department of Obstetrics \& Gynaecology, Safdarjung hospital, Delhi. A total of 60 women, 30 with menorrhagia due to fibroid uterus (Group I) and 30 with menorrhagia due to DUB (Group II) were included in the study. Menstrual blood loss was assessed by the Pictorial Blood Loss Assessment Chart (PBAC) method. The PBAC score was calculated by assigning a score of 1,5 or 20 respectively to a lightly, moderately or fully soaked sanitary pad, and a score of 1 or 5, respectively, for the small or large clots. Inclusion criteria were PBAC score $\geq 100$ and age between 35 and 45 years. Exclusion criteria were uterine size more than 12 weeks, fibroid $\geq 5 \mathrm{~cm}$, fibroid distorting the uterine cavity, any uterine anomaly, any gynaecological malignancy, concurrent or recurrent PID or any systemic illness causing menorrhagia. Approval for the study was obtained from the institutional board and informed consent was taken from each patient before recruiting the subjects for the study.

A detailed history and examination including detailed gynaecological examination was done for every participant. Before recruiting the patients for study, a complete haemogram, serum TSH, Serum Ferritin, Pap smear and endometrial sampling and a transabdominal/ transvaginal USG was carried out for all the patients as part of the usual treatment protocol followed in our hospital. Hysteroscopy was done if an intrauterine lesion was suspected. Once the patient was considered eligible for the study, LNG-IUS was inserted within the first 7 days of menstruation. Patients were instructed and trained to maintain a menstrual calendar and pictorial blood loss assessment chart which was provided to them.

Patients were followed up after 1 week of insertion and then at 1, 3, 6 and 9 months. On each follow up visit, patients' subjective assessment of decrease in the menstrual blood loss was recorded.

Semiquantitative assessment of menstrual blood loss was done by PBAC score. Change in the number of 'bleeding days' was also noted. The patients were also inquired about any change in the menstrual patterns, symptoms of dysmenorrhea and any known side effects. Transvaginal sonography (TVS) was also done at each visit to confirm the position of LNG-IUS and to assess uterine volume, myoma volume, endometrial thickness, and the distance from the distal portion of LNG-IUS to uterine fundus.

At the end of the study, patient satisfaction with the treatment was also assessed by using a 5-level questionnaire. The patients were asked to rate their satisfaction with the treatment on a scale of five, ranging from 'very satisfied' to 'not at all satisfied'. Failure to treatment was defined as persistent menorrhagia, or expulsion or premature removal due to side-effects or persistent symptoms. Patients with treatment failure were also rated as 'not at all satisfied'.

The statistical analysis was done using "R" statistical package ( $\mathrm{R}$ foundation for statistical computing, Vienna University of Economics and Business, Vienna, Austria). Data concerning patient characteristics were expressed in mean (range). The follow-up data was found to have 
outliers, and hence, non-parametric tests were used for comparative analysis. Median with interquartile range was used for descriptive statistics and Wilcoxon signed rank test was used to compare the data. The cut-off point of $\mathrm{P}$ value $<0.05$ was taken as a level of statistical significance.

\section{RESULTS}

\section{Patient characteristics (Table 1)}

Table 1: Patient characteristics.

\begin{tabular}{|lll|}
\hline Age & $\begin{array}{l}\text { Fibroid group } \\
\text { 40 years (37-44) }\end{array}$ & DUB group \\
\hline BMI & $26.7(22.3-30.4)^{*}$ & $27.8(20-32)^{*}$ \\
\hline Parity & & \\
\hline P1 & $3.3 \%$ & $3.3 \%$ \\
\hline P2 & $60.0 \%$ & $43.3 \%$ \\
\hline P3 & $26.7 \%$ & $43.3 \%$ \\
\hline P4 or above & $10.0 \%$ & $10.0 \%$ \\
\hline Presenting complaints & & \\
\hline Menorrhagia only & $46.7 \%$ & $33.3 \%$ \\
\hline Polymenorrhagia & $30 \%$ & $20 \%$ \\
\hline $\begin{array}{l}\text { Menorrhagia }+ \\
\text { dysmenorrhea }\end{array}$ & $10 \%$ & $23.3 \%$ \\
\hline $\begin{array}{l}\text { Polymenorrhagia }+ \\
\text { dysmenorrhea }\end{array}$ & $13.3 \%$ & $23.3 \%$ \\
\hline Endometrial histology & $36.7 \%$ & $23.3 \%$ \\
\hline Secretory & $40.0 \%$ & $46.7 \%$ \\
\hline $\begin{array}{l}\text { Non-secretory } \\
\text { Simple hyperplasia }\end{array}$ & $23.3 \%$ & $30 \%$ \\
\hline Complex hyperplasia & $0 \%$ & $0 \%$ \\
\hline
\end{tabular}

*median (range)
The median age of the patients was 41.5 years (40 years in the fibroid group and 43 years in the DUB group; range for all patients 36-45 years). The median BMI (with range) of the patients was 26.7 (22.3-30.4) in group I and 27.8 (20-32) in the DUB group. The educational qualification of the majority of the patients was graduate or above.

The baseline PBAC score ranged from 265 to 765 (median 367; IQ range 334.2-550.5) in fibroid patients, and 166 to 897 (median 341.5; IQ range 294.5-564.2) in DUB patients (Tables 2 and 3). The baseline uterine volume ranged from $53.8 \mathrm{cc}$ to $292.3 \mathrm{cc}$ (median 148.5 cc; IQ range 105.0-172.0 cc) in the fibroid group and $48.0 \mathrm{cc}$ to $259.0 \mathrm{cc}$ (median $92.7 \mathrm{cc}$; IQ range 75.6-120.3 cc) in the DUB group.

The most common endometrial histopathological pattern was non-secretory (40\% of fibroid patients and $46.7 \%$ of DUB patients; Table 1). Other patients had secretory pattern or simple hyperplasia. None of the patients had complex hyperplasia.

\section{Follow-up (Tables 2 and 3)}

Out of 60 patients recruited in the study, one patient with DUB was lost to follow-up. A total of 54 patients (90\%) continued the treatment and the follow-up till the end of nine months. Out of the remaining five, four patients underwent premature removal of the device due to persistent menorrhagia (three in fibroid group and one in DUB group). One patient with fibroid had spontaneous expulsion of the device.

Table 2: Follow-up results for patients with fibroid.

\begin{tabular}{|llllll|}
\hline & Baseline & 1 month & 3 months & 6 months & 9 months \\
\hline Number of patients & 30 & 30 & 29 & 27 & 26 \\
\hline PBAC score* & 367 & 171.5 & 87.0 & 27.0 & 8.5 \\
& $(334.2-550.5)$ & $(99.5-391.5)$ & $(24.0-169.0)$ & $(11.0-67.0)$ & $(3.0-25.0)$ \\
\hline P value\# & - & 0.0000 & 0.0000 & 0.0000 & 0.0000 \\
\hline $\begin{array}{l}\text { Subjective \% reduction in MBL as } \\
\text { compared to baseline }\end{array}$ & - & $40 \%(20-65 \%)$ & $70 \%(60-85 \%)$ & $85 \%(77-90 \%)$ & $95 \%(90-95 \%)$ \\
\hline P value\# & - & 0.0000 & 0.0000 & 0.0000 & 0.0000 \\
\hline Number of bleeding days* & $8.5(7.5-9)$ & $5.5(4.5-7.5)$ & $3.5(2.5-4.5)$ & $3.5(2.5-5.5)$ & $2.5(1.5-3.5)$ \\
\hline P value\# & - & 0.0006 & 0.0001 & 0.0001 & 0.0000 \\
\hline Uterine volume (cc)* & 148.5 & 134.1 & 128.4 & 121.6 & 118.4 \\
& $(105.0-172.0)$ & $(113.0-162.6)$ & $(107.0-162.5)$ & $(98.5-159.3)$ & $(100.1-139.1)$ \\
\hline P value\# & - & 0.0020 & 0.0009 & 0.0000 & 0.0008 \\
\hline Endometrial thickness $(\mathrm{mm})$ & $8.1(6.5-11.4)$ & $8.9(6.0-9.2)$ & $7.0(6.0-9.0)$ & $6.2(6.0-8.4)$ & $6.0(5.4-8.0)$ \\
\hline P value\# & - & 0.0970 & 0.0667 & 0.0003 & 0.0000 \\
\hline Myoma volume $(\mathrm{cc})$ & $4.8(2.8-20.2)$ & $3.7(2.7-17.3)$ & $3.7(2.5-18.0)$ & $4.2(2.9-16.5)$ & $4.3(3.0-16.4)$ \\
\hline P value\# & - & 0.1880 & 0.0091 & 0.1482 & 0.2109 \\
\hline Hemoglobin $(\mathrm{g} / \mathrm{dl}) *$ & $10.2(8.9-11.5)$ & $10.6(9.5-11.0)$ & $11.4(10.0-11.6)$ & $11.8(11.5-12.0)$ & $13.0(12.4-13.4)$ \\
\hline P value\# & - & 0.1550 & 0.0003 & 0.0000 & 0.0000 \\
\hline Serum ferritin level (ng/ml)* & $5.5(4.5-6.9)$ & - & - & - & $13.2(11.6-28.8)$ \\
\hline P value\# & - & - & - & - & 0.0000 \\
\hline
\end{tabular}

*Median values (interquartile range); \#P value as compared to the baseline values using Wilcoxon (paired) signed rank test; patients lost to follow-up were excluded for analyses of that particular time point and beyond 
Table 3: Follow-up results for patients with DUB.

\begin{tabular}{|llllll|} 
& Baseline & 1 month & 3 months & 6 months & 9 months \\
\hline Number of patients & 30 & 29 & 29 & 28 & 28 \\
\hline PBAC score* & $341.5(294.5-564.2)$ & $115.0(85.0-186.0)$ & $57.0(37.0-89.0)$ & $30.0(9.3-54.0)$ & $17.0(0-28.3)$ \\
\hline P value & - & 0.0000 & 0.0000 & 0.0000 & 0.0000 \\
\hline Subjective \% reduction in MBL & - & $40.0(35.0-50.0)$ & $70.0(55.0-80.0)$ & $85.0(75.0-86.3)$ & $90.0(80.0-100)$ \\
\hline P value & - & 0.0000 & 0.0000 & 0.0000 & 0.0000 \\
\hline Number of bleeding days* & $7.5(6.5-11)$ & $5.5(4.5-9)$ & $4.5(2.5-6.5)$ & $3.25(2.25-4.5)$ & $2.5(0-3.5)$ \\
\hline P value & - & 0.065 & 0.0000 & 0.0000 & 0.0000 \\
\hline Uterine volume (cc)* & $92.7(75.6-120.3)$ & $93.8(60.5-115.8)$ & $90.6(57.4-107.3)$ & $76.3(53.4-94.9)$ & $73.3(50.9-89.8)$ \\
\hline P value $^{\#}$ & - & 0.0543 & 0.0005 & 0.0000 & 0.0000 \\
\hline Endometrial thickness (mm) $^{\#}$ & $11.5(9.6-16.0)$ & $10.0(9.8-12.0)$ & $9.5(8.0-11.0)$ & $9.0(7.6-10.5)$ & $8.0(6.5-9.0)$ \\
\hline P value $^{\#}$ & - & 0.0003 & 0.0000 & 0.0000 & 0.0000 \\
\hline Hemoglobin $(\mathrm{g} / \mathrm{dl}) *^{*}$ & $10.6(10.1-11.0)$ & $11.0(10.0-11.0)$ & $11.0(11.0-11.6)$ & $11.6(11.2-12.0)$ & $12.0(11.9-12.4)$ \\
\hline P value $^{\#}$ & - & 0.6097 & 0.0000 & 0.0000 & 0.0000 \\
\hline Serum ferritin level $(\mathrm{ng} / \mathrm{ml}) *^{*}$ & $6.8(6.0-10.9)$ & - & - & - & $34.0(31.0-40.5)$ \\
\hline P value $^{\#}$ & - & - & - & - & 0.0000 \\
\hline
\end{tabular}

*Median values (interquartile range); \#P value as compared to the baseline values using Wilcoxon (paired) signed rank test; patients lost to follow-up were excluded for analyses of that particular time point and beyond

\section{Changes in menstrual patterns (Table 4)}

Table 4: Menstrual patterns at baseline and at 9 months follow-up (values in percentages).

\begin{tabular}{|lllll|}
\hline $\begin{array}{l}\text { Menstrual } \\
\text { pattern }\end{array}$ & Baseline & $\begin{array}{l}\text { At 9 } \\
\text { months }\end{array}$ & Baseline & $\begin{array}{l}\text { At 9 } \\
\text { months }\end{array}$ \\
\hline Normal period $^{\mathrm{a}}$ & 0 & 26.9 & 0 & 25.0 \\
\hline Oligomenorrhea $^{\mathrm{b}}$ & 0 & 42.3 & 0 & 50.0 \\
\hline $\begin{array}{l}\text { Intermenstrual } \\
\text { spotting }\end{array}$ & 0 & 15.4 & 0 & 7.1 \\
\hline Amenorrhea $^{\mathrm{c}}$ & 0 & 19.2 & 0 & 28.6 \\
\hline $\begin{array}{l}\text { Persistent } \\
\text { menorrhagia }\end{array}$ & 100 & 11.1 & 100 & 0 \\
\hline Dysmenorrhea & 23.3 & 15.4 & 46.7 & 10.7 \\
\hline Polymenorrhea $^{\mathrm{d}}$ & 43.3 & 0 & 43.3 & 0 \\
\hline
\end{tabular}

${ }^{a}$ Normal cyclical bleeding but with normal blood flow

${ }^{\mathrm{b}}$ Oligomenorrhea defined as bleeding at intervals $>45$ days

${ }^{\mathrm{c}}$ Amenorrhea defined as an absence of bleeding for atleast 3 months

${ }^{\mathrm{d}}$ Polymenorrhea defined as bleeding at interval $<21$ days

Fibroid group: Eighteen out of 26 patients (69.2\%) had normal periods or oligomenorrhea at 9 months follow-up. Five patients developed amenorrhea. Only three patients had persistent menorrhagia at 9 months. Including the four patients who had premature removal or expulsion, the failure rate for the treatment was $23.3 \%$ ( 7 out of 30 ).

No patient had polymenorrhea at 9 months. Dysmenorrhea decreased from $23.3 \%$ at baseline to $15.4 \%$ at final follow-up. Some patients however developed intermenstrual spotting during the initial months which persisted in $15.4 \%$ till the last follow-up.
DUB group: Twenty one out of 28 patients $(75 \%)$ had normal periods or oligomenorrhea at 9 months follow-up. Eight patients developed amenorrhea. None of the patients followed up till 9 months had persistent menorrhagia or polymenorrhea. Only one patient had persistent menorrhagia who underwent premature removal. Thus, the treatment failure rate was $3.4 \%$ in DUB group (1 out of 29 patients). Dysmenorrhea also decreased from $46.7 \%$ at baseline to $10.7 \%$ at final follow-up. As with the fibroid group, some patients developed intermenstrual spotting (17.1\% at 9 months).

The patients who had premature removal or expulsion were excluded from subsequent follow-up assessments after the removal or expulsion as they underwent hysterectomy.

\section{Changes in menstrual blood loss (Figure 1)}

Progressive reduction in menstrual blood loss was observed, which was statistically significant even as early as 1 month in both the groups as compared to the baseline. At 9 months, in fibroid patients, the median PBAC score reduced to 8.5 (IQ range 3.0-25.0), a 97.7\% reduction as compared to baseline $(\mathrm{P}=0.000)$. DUB patients also had a $95 \%$ reduction in median PBAC score to 17.0 at 9 months (IQ range $0-28.3 ; \mathrm{P}=0.000$ ).

Patients reported a median $40 \%$ subjective reduction in blood loss as early as 1 month after the insertion in both groups, which was statistically significant. At 9 months, the fibroid patients felt a median 95\% reduction in menstrual blood loss (MBL) as compared to baseline (IQ range $90-95 \%$ ). DUB patients reported a median $90 \%$ reduction in MBL at 9 months (IQ range 80-100\%). 


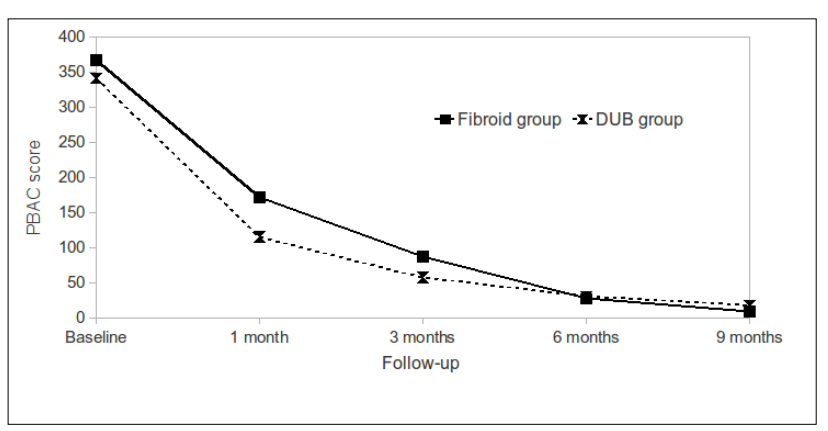

Figure 1: PBAC scores over the follow-up period in DUB patients and fibroid patients.

\section{Change in uterine volume}

There was a progressive reduction in the mean uterine volume in the fibroid group starting from the 1st followup visit ( $\mathrm{P}<0.05$ at all visits). At the end of 9 months the uterine volume had decreased significantly from a median of $148.5 \mathrm{cc}$ at baseline to $118.4 \mathrm{cc}(\mathrm{P}=0.000)$. The volume of the fibroid was also found to decrease initially but it returned to near-baseline values at final follow-up.

The patients with DUB also had a significant reduction in the median uterine volume, from $92.7 \mathrm{cc}$ at baseline to $73.3 \mathrm{cc}$ at 9 months $(\mathrm{P}$ value $=0.000)$.

\section{Change in haemoglobin and transferrin levels}

The median haemoglobin concentration increased significantly from 3 months onwards in both the groups. At the end of the study, the median haemoglobin concentration increased by $2.8 \mathrm{~g} / \mathrm{dl}$ in the fibroid group and by $1.4 \mathrm{~g} / \mathrm{dl}$ in DUB group $(\mathrm{P}=0.000)$. The serum ferritin levels increased by $140 \%$ in fibroid group and by $400 \%$ in DUB group $(\mathrm{P}=0.000)$.

\section{Side effects}

There was no major side effect in both the groups. Water retention was the most common side effect (17 patients, both groups combined). Other side effects included weight gain (11 patients), breast engorgement (9 patients), lower abdominal discomfort (9 patients) and mood changes (1 patient). Seven patients were found to develop ovarian cyst during the period of study. Serum FSH \& LH levels were done at the beginning and at the end of study and no alteration in their levels was observed.

\section{Patient satisfaction (Figure 2)}

The five patients who had premature removal of the LNG-IUS due to persistent menorrhagia or spontaneous expulsion were 'not at all satisfied' as expected. All of them had subsequent hysterectomy. Most of the patients in both the groups (20 out of 30 in fibroid group and 24 out of 29 in DUB group) were either 'very satisfied' or 'satisfied' with the treatment.

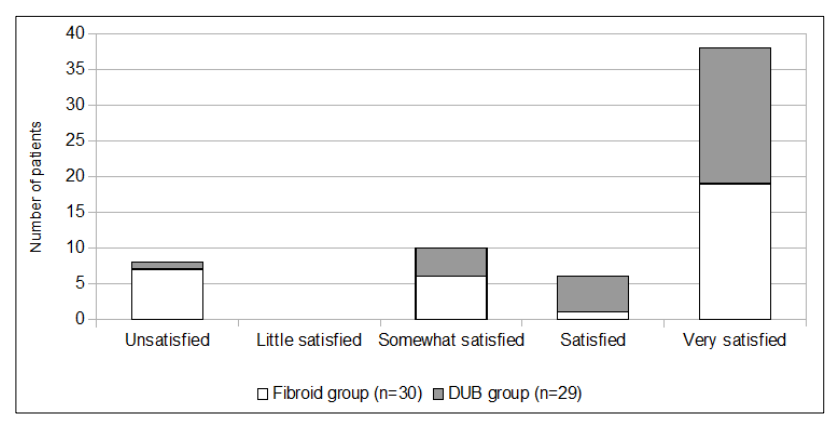

Figure 2: Patient satisfaction at 9 months (or at the time of premature removal) as assessed on a five-point scale (absolute number of patients). White area represents fibroid patients and shaded grey area represents DUB patients. Majority of patients were either "very satisfied" or "satisfied".

\section{DISCUSSION}

We report good results with LNG-IUS for the treatment of DUB and fibroid. In DUB patients, with 9 months follow-up, the treatment failure rate was only $3.4 \%$ (1 out of 29 patients), and the median PBAC score reduced by $95 \%$ at 9 months. Subjectively also, patients reported reduction in blood loss starting as early as 1 month after the insertion, which was statistically significant. At 9 months, the DUB patients reported a median $90 \%$ reduction in MBL, and $82.8 \%$ of the patients were either 'very satisfied' or 'satisfied' with the treatment.

Fibroid patients also had an equally impressive $97.7 \%$ reduction of PBAC score at 9 months, although the failure rate was higher in patients of fibroid $(23.3 \% ; 7$ out of 30). Patients reported statistically significant subjective reduction in blood loss from the first month, and at 9 months, there was a median $95 \%$ reduction in menstrual blood loss (MBL). Majority of patients were either 'very satisfied' or 'satisfied' with the treatment, although the percentage of such patients (66.7\%) was less than that in the DUB group.

Haemoglobin values and serum ferritin levels significantly increased in both groups. No major side effect was noted in any of the groups.

Other studies have also previously reported good results with LNG-IUS in patients of DUB. Chattopdhyay et al. prospectively followed up 42 women with idiopathic menorrhagia treated with LNG-IUS, and reported significant reduction in mean blood loss. ${ }^{10}$ In another recent study by Xu et al., $83.5 \%$ of patients of DUB were very satisfied or satisfied with the results of LNG-IUS, compared to only $59.2 \%$ of patients treated with conventional medical treatment like contraceptive pills, oral progestins, etc. ${ }^{16}$ These satisfaction rates are comparable to the findings in our study $(82.8 \%$ patients 
very satisfied or satisfied). A similar study comparing LNG-IUS with oral norethisterone reported $90 \%$ patient satisfaction with LNG-IUS, as against $20 \%$ with norethisterone. ${ }^{17}$ LNG-IUS has been shown to have blood loss reduction and patient satisfaction rates comparable to those after trans-cervical endometrial resection. ${ }^{18}$ Given the fact that LNG-IUS requires less skills, is much less invasive, and has no operative risks, this should be the treatment of choice for DUB.

Some previous studies have shown effectiveness of LNGIUS in fibroid also, although the results have not been as impressive as in DUB patients, and in fact, some reports have shown no benefit of LNG-IUS. For example, in a study of 19 patients by Mercorio et al., the menstrual pattern changed to non-menorrhagic in only five out of 32 patients (even though overall there was a $69 \%$ reduction in the PBAC scores). ${ }^{18}$ In their study, the device was prematurely removed (removal due to menorrhagia or spontaneous expulsion) in 11 patients, and 14 other patients had persistent menorrhagia. Their inclusion criteria were more or less similar to ours.

Other studies have shown better results. Kriplani et al reported $97.4 \%$ reduction in PBAC scores in myoma patients at 12 months with significant reduction of mean uterine volume. ${ }^{19}$ However, similar to the findings in our study, they did not find any significant reduction in the volume of the myoma per se. Other studies have found satisfactory outcomes with LNG-IUS for fibroids less than $2.5 \mathrm{~cm}$, but not for those measuring more than $2.5 \mathrm{~cm} .^{20}$

Another advantage of LNG-IUS is contraception. In a study of 30 fibroid patients receiving LNG-IUS with the primary aim of contraception, no pregnancy was reported during the 12 months follow-up period. ${ }^{21}$ Significant reduction in blood loss and improvement in haemoglobin levels was reported in their study, and there was only one expulsion. There were however, no changes in both uterine as well as myoma volumes.

For perimenopausal patients, hysterectomy is considered the most effective treatment option for symptomatic fibroids. ${ }^{22}$ However, LNG-IUS is a valid alternative for patients wanting to avoid hysterectomy. In a recent study by Machado et al, not only did the treatment with LNGIUS help avoid hysterectomy in $89.5 \%$ of cases, but also, the patients treated with LNG-IUS were more satisfied compared to those primarily undergoing hysterectomy. ${ }^{9}$

While hysteroscopic myomectomy is a good option for submucosal fibroids, the surgical risks of myomectomy become more relevant in non-submucosal fibroids, and such cases presenting with menorrhagia can be better dealt with LNG-IUS. Thermal ballon ablation is another option for such patients, which has been shown to have similar success rates $(76 \%)$ as with LNG-IUS (79\%). ${ }^{20}$ However, thermal balloon ablation is definitely a more invasive procedure with greater risks as compared to LNG-IUS which is much safer.

\section{CONCLUSION}

In conclusion, LNG-IUS is an excellent treatment modality for patients of DUB, with reduction in blood loss, improvement in haemoglobin and ferritin levels, and good patient satisfaction. It is also a useful treatment option in non-submucosal small fibroids, particularly for the symptoms of menorrhagia, can reduce uterine volume and can help avoid hysterectomy, but there is no effect on fibroid volume. Majority of patients are satisfied, although satisfaction rates are less than those observed in DUB patients.

\section{Funding: No funding sources}

Conflict of interest: None declared

Ethical approval: The study was approved by the institutional ethics review board

\section{REFERENCES}

1. Hallberg L, Högdahl AM, Nilsson L, Rybo G. Menstrual blood loss: a population study. Variation at different ages and attempts to define normality. Acta Obstet Gynaecol Scand. 1966;45(3):320-51.

2. Shaw RW. Assessment of medical treatments for menorrhagia. $\mathrm{Br} \quad \mathrm{J}$ Obstet Gynaecol. 1994 Jul;101(Suppl 11):15-8.

3. Stewart EA. Uterine fibroids. Lancet. 2001 Jan;357(9252):293-8.

4. Stirrat GM. Choice of treatment for menorrhagia. Lancet. 1999 Jun;353(9171):2175-6.

5. Vuorma S, Teperi J, Hurskainen R, Keskimäki I, Kujansuu E. Hysterectomy trends in Finland in 1987-1995: a register based analysis. Acta Obstet Gynaecol Scand. 1998 Aug;77(7):770-6.

6. Hubacher D, Grimes DA. Non-contraceptive health benefits of intrauterine devices: a systematic review. Obstet Gynaecol Surv. 2002 Feb;57(2):120-8.

7. Hurskainen R, Teperi J, Rissanen P, Aalto A-M, Grenman S, Kivelä A, et al. Clinical outcomes and costs with the levonorgestrel-releasing intrauterine system or hysterectomy for treatment of menorrhagia: randomized trial 5-year follow-up. JAMA. 2004 Mar;291(12):1456-63.

8. Rauramo I, Elo I, Istre O. Long-term treatment of menorrhagia with levonorgestrel intrauterine system versus endometrial resection. Obstet Gynaecol. 2004 Dec;104(6):1314-21.

9. Machado RB, de Souza IM, Beltrame A, Bernardes CR, Morimoto MS, Santana N. The levonorgestrelreleasing intrauterine system: its effect on the number of hysterectomies performed in perimenopausal women with uterine fibroids. Gynecol Endocrinol. 2013 May;29(5):492-5.

10. Chattopdhyay B, Nigam A, Goswami S, Chakravarty PS. Clinical outcome of levonorgestrel intra-uterine system in idiopathic menorrhagia. Eur Rev Med Pharmacol Sci. 2011 Jul;15(7):764-8. 
11. Luukkainen $\mathrm{T}$, Lähteenmäki $\mathrm{P}$, Toivonen $\mathrm{J}$. Levonorgestrel-releasing intrauterine device. Ann Med. 1990 Apr;22(2):85-90.

12. Lähteenmäki $P$, Rauramo I, Backman $T$. The levonorgestrel intrauterine system in contraception. Steroids. 2000 Nov;65(10-11):693-7.

13. Sivin I, el Mahgoub S, McCarthy T, Mishell DR, Shoupe D, Alvarez F, et al. Long-term contraception with the levonorgestrel $20 \mathrm{mcg} / \mathrm{day}(\mathrm{LNg} 20)$ and the copper T 380Ag intrauterine devices: a five-year randomized study. Contraception. 1990 Oct;42(4):361-78.

14. Jones RL, Critchley HO. Morphological and functional changes in human endometrium following intrauterine levonorgestrel delivery. Hum Reprod. 2000 Aug;15(Suppl 3):162-72.

15. Grigorieva V, Chen-Mok M, Tarasova M, Mikhailov A. Use of a levonorgestrel-releasing intrauterine system to treat bleeding related to uterine leiomyomas. Fertil Steril. 2003 May;79(5):1194-8.

16. $\mathrm{Xu} \mathrm{L}$, Lee BS, Asif $\mathrm{S}$, Kraemer $\mathrm{P}$, Inki $\mathrm{P}$. Satisfaction and health-related quality of life in women with heavy menstrual bleeding; results from a non-interventional trial of the levonorgestrelreleasing intrauterine system or conventional medical therapy. Int J Womens Health. 2014;6:547-54.

17. Naqaish T, Rizvi F, Khan A, Afzal M. Patient satisfaction for levonorgestrel intrauterine system and norethisterone for treatment of dysfunctional uterine bleeding. J Ayub Med Coll Abbottabad. 2012 Mar;24(1):23-6.
18. Mercorio F, De Simone R, Di Spiezio Sardo A, Cerrota G, Bifulco G, Vanacore F, et al. The effect of a levonorgestrel-releasing intrauterine device in the treatment of myoma-related menorrhagia. Contraception. 2003 Apr;67(4):277-80. Gupta B, Mittal S, Misra R, Deka D, Dadhwal V. Levonorgestrel-releasing intrauterine system vs. transcervical endometrial resection for dysfunctional uterine bleeding. Int J Gynaecol Obstet. 2006 Dec;95(3):261-6.

19. Kriplani A, Awasthi D, Kulshrestha V, Agarwal N. Efficacy of the levonorgestrel-releasing intrauterine system in uterine leiomyoma. Int J Gynaecol Obstet. 2012 Jan;116(1):35-8.

20. Kim JY, No JH, Kim K, Kim YB, Jee BC, Lee JR, et al. Effect of myoma size on failure of thermal balloon ablation or levonorgestrel releasing intrauterine system treatment in women with menorrhagia. Obstet Gynaecol Sci. 2013 Jan;56(1):36-40.

21. Xie Z-W, Zhang Y-N, Wan S, Xu W-Z, Chen J. Levonorgestrel-releasing intrauterine device is an efficacious contraceptive for women with leiomyoma. J Int Med Res. 2012;40(5):1966-72.

22. Marret H, Fritel X, Ouldamer L, Bendifallah S, Brun J-L, De Jesus I, et al. Therapeutic management of uterine fibroid tumors: updated French guidelines. Eur J Obstet Gynecol Reprod Biol. 2012 Dec;165(2):156-64.

DOI: 10.5455/2320-1770.ijrcog20140960

Cite this article as: Gupta R, Dewan R, Mittal P, Suri J, Dewan A, Bharti R. Role of levonorgestrel releasing intra-uterine system in the treatment of menorrhagia due to dysfunctional uterine bleeding and fibroid uterus. Int J Reprod Contracept Obstet Gynecol 2014;3:671-7. 